\title{
OPTICAL NONLINEARITIES IN BULK GaAs DETERMINED BY EL2 DEFECT
}

\author{
M. Sudzius, L. Bastiene, S. Svitojus and K. Jarasiunas \\ Institute of Material Science and Applied Research, Division of Optical Diagnostics \\ Vilnius University \\ Sauletekio a ve 9-3, 2054 Vilnius, Lithuania
}

Time-resolved studies of light diffraction on free carrier phase gratings and light absorption in subnanosecond time domain were carried out in two distinct areas of semi-insulating $\mathrm{GaAs}$ with high and low growth-defect density. Numerical analysis was performed in order to reveal the role of EL2 defect in carrier generation and transport. The possibility of transient grating technique to study various defect-governed carrier relaxation processes were demonstrated experimentally.

PACS numbers: $72.20 . \mathrm{Jv}$

Optical illumination of GaAs:EL2 at $1.06 \mu \mathrm{m}$ leads to the change of its complex refractive index via free carrier (FC) nonlinearity, described by the DrudeLorentz model. Modulation of refractive index $\Delta n$ is governed by non-equilibrium carrier concentration $\Delta N, \Delta n \approx-n_{\mathrm{eh}} \Delta N$, where $n_{\mathrm{eh}} \approx 2 \times 10^{-21} \mathrm{~cm}^{-3}$ is refractive index modulation by one electron-hole pair, and absorption index modulation is due to free carriers and transformation of defect charge state. In a case of spatially modulated light interference pattern, the electrical and optical properties of medium are spatially modulated as well.

The solution of continuity equation yields the dynamics and simple relationship between the modulating FC concentration $\Delta N(t)=N_{\max }(t)-N_{\min }(t)$ and optically measured values

$$
\Delta N(t)=2 \alpha(1-R) I_{0} \int_{0}^{t} f(t-\xi) \exp \left(\frac{-\xi}{\tau_{\mathrm{e}}}\right) \mathrm{d} \xi,
$$

where $\alpha, R$ are absorption and reflection coefficients, $I_{0}$ is the incident beam intensity, $f(t)$ describes the temporal shape of the laser pulse. The grating decay time $\tau_{\mathrm{e}}$ is governed by recombination and diffusion $1 / \tau_{\mathrm{e}}=1 / \tau_{\mathrm{r}}+1 / \tau_{\mathrm{d}}$. The rate of diffusion decay can be monitored by changing the grating period $\Lambda=\lambda / \sin \theta=$ $2 \pi\left(\tau_{\mathrm{d}} D\right)^{1 / 2}$, where $\theta$ is the angle between interfering beams, and $D$ is the diffusion coefficient. Hence, measurements of grating decay time at different $\Lambda$ enable one to separate the erasure processes. 
The diffracted signal $I_{1}$ integrates and amplifies the carrier modulation depth over the sample thickness. Its ratio to transmitted probe beam intensity $I_{\mathrm{T}}$, called diffraction efficiency of the grating, in the simplest case is the following:

$$
\eta=\frac{I_{1}}{I_{T}}=\left(\frac{\pi \Delta n d}{\lambda}\right)^{2}
$$

where $\Delta n, d$ are respectively refractive index variation and the thickness of the crystal.

The experiments were performed by using 30 ps duration YAG-laser pulses in degenerate four wave mixing configuration (DFWM), i.e. the weak probe beam was scattered from a transient grating induced in crystal by two, crossed at angle $\theta$, much stronger recording beams. The power of all beams was monitored during the experiment. In case when one of recording beams was closed we realized probe-beam technique. The details of the experimental setups can be found elsewhere [1]. The samples used in the experiments were LEC-grown semi-insulating (SI) In-alloyed GaAs wafers with different density of EL2.

Optical and electrical properties of undoped SI LEC-grown GaAs for a near-IR wavelengths are caused by intrinsic EL2 donor located at $0.75 \mathrm{eV}$ below the bottom of conduction band. The Fermi level is pinned at this level, thus EL2 is both in neutral EL2 ${ }^{\circ}$ and ionized state EL2 ${ }^{+}$[2]. Since our measurements are done at room temperature, we shall not be concerned with the metastable state EL2* due to its very efficient thermal regeneration to neutral EL2 state [3]. Then the total absorption coefficient can be written as

$$
\alpha=\sigma_{n}^{0} N^{0}+\sigma_{p}^{0} N^{+}+\alpha_{0},
$$

where $\sigma_{n}^{0}, \sigma_{p}^{0}$ are optical absorption cross-sections for neutral $\left(N^{0}\right)$ and ionized $\left(N^{+}\right)$EL2 sites respectively, while $\alpha_{0}$ is responsible for any absorption process, unrelated to EL2 (e.g. two-photon absorption).

We performed theoretical analysis of carrier generation and transport at 30 ps pulse excitation and $\Lambda=5 \mu \mathrm{m}$. Concentration of free carriers (electrons and holes), ionized deep levels and space-charge (SC) electric field dynamics follow from the solution of continuity equations for electrons, holes, ionized EL2 densities and Poisson's equation [4]. In the given range of excitations, varying from $0.05 \mu \mathrm{J} / \mathrm{cm}^{2}$ to $5 \mathrm{~mJ} / \mathrm{cm}^{2}$, refractive index modulation $\Delta n=2 \times 10^{-9}-7 \times 10^{-3}$ are reached at the end of the laser pulse (Fig. 1a). At the lowest excitations the linear carrier generation from deep traps dominates: $N \gg P(N, P$ are electron and hole concentration respectively) and FC decay approaches monopolar diffusion time $\tau_{\mathrm{e}}=(\Lambda / 2 \pi)^{2}\left(e / k_{\mathrm{B}} T \mu_{n}\right) \approx 50 \mathrm{ps}$. During the diffusive decay of this grating, a SC electric field component between mobile carriers (electrons) and ionized donors develops and approaches its diffusion limit $E_{\mathrm{SC}}=(2 \pi / \Lambda)\left(k_{\mathrm{B}} T / e\right) \approx 310 \mathrm{~V} / \mathrm{cm}$. Thus, the electrons drift current opposes the diffusion current, and the further decay of both gratings is governed by carrier recombination with $\tau_{\mathrm{e}}=1 / \gamma_{n} N^{+}$, where $\gamma_{n}$ is the electron recombination coefficient in the deep trap. With increasing excitation hole generation rate approaches the electron one: density of EL2 traps, responsible for electron generation saturates, but the increasing fluence will 

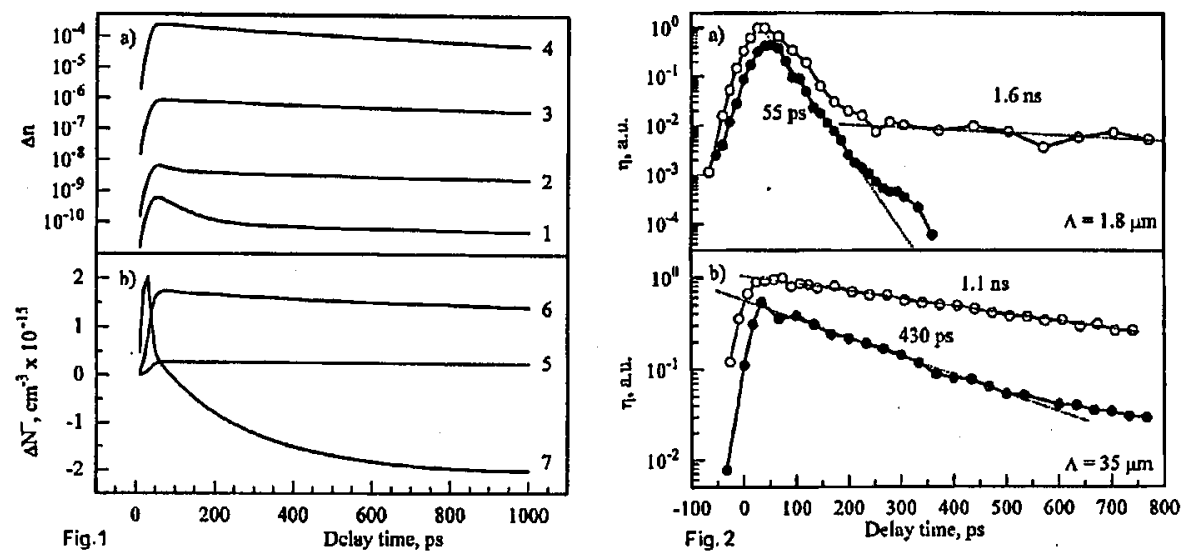

Fig. 1. Calculated time dependencies of refractive index modulation by free carriers (a) and ionized EL2 modulation (b) at grating period $5 \mu \mathrm{m}$ and different excitation levels: $0.05 \mu \mathrm{J} / \mathrm{cm}^{2}$ (1), $0.5 \mu \mathrm{J} / \mathrm{cm}^{2}(2), 50 \mu \mathrm{J} / \mathrm{cm}^{2}(3,5), 0.5 \mathrm{~mJ} / \mathrm{cm}^{2}(6), 5 \mathrm{~mJ} / \mathrm{cm}^{2}(4,7)$. Fig. 2. Free carrier grating decay in area with high (open points) and low (full points) density of EL2 defect at grating period $1.8 \mu \mathrm{m}$ (a) and $35 \mu \mathrm{m}(\mathrm{b})$.

compensate this channel of carrier generation by more efficient two-photon and two-step transitions through deep level. The initial diffusive decay corresponds to carrier effective mobility $\mu=(N+P) /\left(N / \mu_{p}+P / \mu_{n}\right)$, which will reach the limit of pure ambipolar mobility $\mu_{\mathrm{a}}$ at $N=P$ and corresponding Dember SC electric field. In addition, transmittivity studies may reveal different processes of deep defect charge state transformation, leading to bleaching and induced absorption (Fig. 1b).

This carrier transport model is in good agreement with experimental data of time-resolved diffraction (Fig. 2). For small grating periods, when condition $\tau_{\mathrm{d}} \ll \tau_{\mathrm{r}}$ is fulfilled, the diffusive erasure mechanism of transient grating dominates (see Fig. 2a). In the case of high EL2 concentration, grating decay is slower; we attribute the last component of the grating decay with $\tau_{\mathrm{e}} \approx 1.6 \mathrm{~ns}$ to the effect of light created SC field and carrier drift. If concentration of EL2 is small, SC field is either insufficient to slower carrier diffusion, or it has Dember origin. At large grating period (Fig. $2 \mathrm{~b}$ ) $\tau_{\mathrm{r}} \ll \tau_{\mathrm{d}}$, thus recombination processes dominate. In high EL2 density area one clearly can see single-exponential decay with $\tau_{\mathrm{e}}=\tau_{\mathrm{r}} \approx 1.1 \mathrm{~ns}$, which is larger than that in low EL2 density area with $\tau_{\mathrm{r}} \approx 430 \mathrm{ps}$. We attribute the decay times to the band-gap modulation by charged dislocations. The local region around dislocations (up to $10 \mu \mathrm{m}$ ) is perturbed: inhomogeneous stress field and electric field accompany the dislocations, which in SI GaAs are charged by electrons from EL2 centers [5]. Such kind of local space charge can significantly modify band-gap, thus influence carrier spatial separation and their recombination [6].

Calculation of absorption coefficient modulation shows that an amplitude grating may be created in EL2 traps due to their spatial photoionization [1]. Thus, both free carrier phase grating and amplitude grating may lead to slow, 


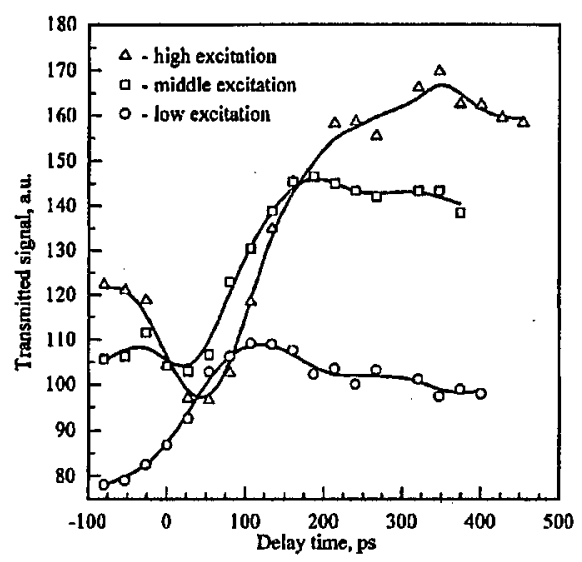

Fig. 3. Time-resolved transmittance in high EL2 density area at different excitation levels.

recombination governed grating decay, which is seen experimentally. In the given paper, free carrier absorption is not taken into account due to low concentrations of non-equilibrium carriers.

Time-resolved studies of light absorption revealed processes of bleaching (at low excitations) and temporal darkening (Fig. 3). These effects can be analyzed using numerical modeling of deep trap modification (see Fig. 1b). Decreasing with excitation density of neutral EL2 ${ }^{0}$ leads to bleaching (if $N<N^{0}$ ), while the fast recombination may reverse the initial trap filling at high excitations (if $N>N^{+}$, see curve 7).

In conclusion, time-resolved studies of refractive and absorptive index revealed light-induced mechanisms of EL2 charge state transformation and its dynamics at room temperature. The simultaneous studies of refractive index modulation by SC electrical field (photorefractive gratings) will provide more detailed information on charge transport, and origin and strength of SC fields.

\section{References}

[1] K. Jarasiunas, Ph. Delaye, G. Roosen, Phys. Status Solidi B 175, 445 (1993).

[2] G.A. Baraf, M.A. Shulter, Phys. Rev. B 45, 4515 (1992).

[3] M. Sudzius, L. Bastiene, K. Jarasiunas, SPIE 2648, 232 (1995).

[4] Ph. Delaye, L. Bastiene, K. Jarasiunas, G. Roosen, SPIE 2097, 474 (1993).

[5] J.-L. Farvacque, B. Podor, Phys. Status Solidi B 167, 687 (1991).

[6] J. Vaitkus, E. Gaubas, K. Jarasiunas, M. Petrauskas, Semicond. Sci. Technol. 7, A131 (1992). 